\title{
Molecular detection methods and serotyping performed directly on clinical samples improve diagnostic sensitivity and reveal increased incidence of invasive disease by Streptococcus pneumoniae in Italian children
}

\author{
Chiara Azzari, Maria Moriondo, Giuseppe Indolfi, Cristina Massai, \\ Laura Becciolini, Maurizio de Martino and Massimo Resti
}

Correspondence

Chiara Azzari

chiara.azzari@unifi.it

Received 2 February 2008

Accepted 22 April 2008

\author{
Department of Paediatrics, University of Florence and Anna Meyer Children's Hospital, Viale \\ Pieraccini 24, Florence 1-50139, Italy
}

\begin{abstract}
The aims of this study were to evaluate the incidence of invasive pneumococcal disease (IPD) in Italian children and perform serotyping by PCR-based assays directly on clinical samples. A 1year paediatric (0-14 years) population-based surveillance study was designed to evaluate the incidence of IPD in the province of Florence, Italy, by cultural and molecular methods. Among 92 children (80 with pneumonia, 8 with meningitis/sepsis, 4 with arthritis), 4 cases of IPD were diagnosed both by culture and real-time PCR and 18 cases exclusively by molecular methods. The sensitivity of molecular methods was significantly higher than that of cultural methods (Cohen's $\kappa$ 0.41; McNemar $P=0.000008$ ). The incidence of IPD in children below 2 years of age was $11.5 / 100000$ and $51.8 / 100000$ by cultural and molecular methods, respectively.

Pneumococcal serotyping by multiplex sequential PCR was obtained in 19/22 samples. Real-time PCR and multiplex sequential PCR can be used directly on biological samples, improving the ability to diagnose IPD. The incidence of IPD appears 5-10 times higher by PCR than by cultural methods.
\end{abstract}

\section{INTRODUCTION}

Streptococcus pneumoniae is a major aetiological agent for pneumonia, meningitis, otitis and sepsis, among both young children and elderly people all over the world (Black et al., 2007; CDC, 1997). The organism is the cause of at least 1.2 million deaths each year in children due to sepsis, meningitis and pneumonia (CDC, 1997; Hausdorff et al., 2000).

The incidence of invasive pneumococcal disease (IPD) varies across countries (Domínguez et al., 2002; Smith et al., 1998; Whitney et al., 2003) and is probably largely underestimated since diagnosis of invasive pneumococcal infections, usually performed by culturing from whole blood or cerebrospinal fluid (CSF), requires the presence of viable pathogens in the clinical samples. Very few data are available for Italy, where the only available source is a passive surveillance system started in 1994 (National Surveillance System for Bacterial Meningitis) where both meningitis and sepsis are reported (Italian National Institute of Health, 2007; http://www.simi.iss.it/dati.htm). According to that system, the mean incidence rate for

Abbreviations: CSF, cerebrospinal fluid; IPD, invasive pneumococca disease; RT, real-time. meningitis seems to be around 0.5/100000 (Italian National Institute of Health, 2007; http://www.simi.iss. it/dati.htm). A prospective surveillance of IPD was undertaken in two Italian regions in 2001 by standard cultural methods and the highest incidence recorded for children was 6-11/100 000 for children below 2 years of age (D’Ancona et al., 2005).

PCR-based assays for direct detection of pneumococci from clinical samples do not require viable bacteria and reach a very high sensitivity, representing an important tool in the diagnosis of invasive pneumococcal infections (Clarke, 2006). However, up to now, PCR-based methods on clinical samples have been used to discriminate between pneumococci and other pathogens (Corless et al., 2001) but not for pneumococcal serotyping. Serotype distribution is usually monitored by serological determination of the capsular type by standard capsular tests after culture (D'Ancona et al., 2005) or by molecular serotyping of isolates (Pai et al., 2006; Kong et al., 2006), so that pneumococcal serotyping cannot be performed on culturenegative samples.

The aim of the present study was to evaluate the incidence of IPD through a 1-year paediatric population-based 
surveillance study in an Italian province and to perform pneumococcal serotyping using molecular methods directly on clinical samples.

\section{METHODS}

Study design. An observational, prospective, cohort study was designed to evaluate the incidence of IPD in a paediatric population in the province of Florence, Italy, in a 12-month period between 1 December 2005 and 30 November 2006. The study was performed using a molecular diagnostic method, set up in the Laboratory of Paediatric Immunology of the University of Florence, directly on biological samples.

Patients. All children 0-14 years referred to the Anna Meyer Children's Hospital were considered. The Anna Meyer Children's Hospital is the only hospital in the district of Florence in which a paediatric infectious disease unit and a paediatric intensive care unit are present, and it is the only one in the province where patients with meningitis, sepsis, arthritis or osteomyelitis as well as with complicated pneumonia are admitted. The Anna Meyer Children's Hospital is also a third level hospital and accepts patients from all of Tuscany and other Italian regions. Thus, in order to avoid overestimation of pneumococcal incidence, all analyses were uniquely calculated on the basis of the cohort of residents in the district of Florence (Italian National Institute of Statistics, ISTAT, 2007; http://demo.istat.it/). All Florentine children with fever, leukocytosis and/or increased levels of serum C-reactive protein and a clinical diagnosis of meningitis, sepsis, complicated pneumonia, osteomyelitis or arthritis were included in the study. Sepsis was clinically suspected by the presence of previously described signs and confirmed by blood tests (Russell, 2006). Meningitis was clinically suspected by the presence of a compatible clinical syndrome and confirmed by chemical (Overturf, 2005) and microbiological CSF tests (cultural or molecular testing). Osteomyelitis or arthritis were clinically suspected by the presence of local signs (Kaplan, 2005) and confirmed by radiological procedures. Complicated pneumonia was suspected in the presence of clinical signs such as tachypnoea and pathological breath sounds (Ostapchuk et al., 2004) and confirmed by X-ray consistent with pneumonia and demonstrating lobar or segmental lung consolidation with or without pleural effusion. An IPD case was defined as one in a child admitted to Anna Meyer Children's Hospital in the study period with one of the above-mentioned diseases and a laboratory-confirmed presence of S. pneumoniae in whole blood and/ or CSF and/or pleural fluid by cultural and/or molecular tests. Clinical conditions preceding the present episode, previous vaccinations and any underlying disease were recorded. Informed consent was obtained for their child by parents or guardians.

Data sources. Data concerning children $0-14$ years, resident in the district of Florence, were provided by the National Institute of
Statistics (Italian National Institute of Statistics, ISTAT, 2007; http:// demo.istat.it/).

Vaccination. In the province of Florence, as well as in the whole Tuscan region, pneumococcal vaccination is not included in the vaccination schedule for the paediatric or adult population. Conjugate vaccine is sometimes suggested by individual paediatricians mainly for infants attending day-care centres; vaccination expenses (both vaccine and administration expenses) are paid by the families with a co-payment system.

Clinical samples. Whole blood was obtained from all children included in the study; CSF was obtained from children with a clinical suspicion of meningitis. Pleural fluid was obtained from two patients who needed a pleural drainage. Biological samples were obtained as soon as possible after hospital admission and used both for cultural testing and for molecular testing by real-time (RT)-PCR and multiplex sequential PCR.

DNA extraction. Bacterial genomic DNA was extracted from $200 \mu \mathrm{l}$ of biological samples using the QIAmp DNeasy Blood \& Tissue kit (Qiagen), according to the manufacturer's instructions.

RT-PCR. Primers and probes were designed using the ABI Primer Express Software Package based on previously published $\operatorname{ctr} A$, bexA, and $l y t A$ sequences from the meningococcal capsular transfer gene, from the Haemophilus influenzae capsule exporting gene and from the pneumococcal autolysin gene, respectively. The ctrA sequence-specific probe was FAM-labelled, the bexA probe was NED-labelled, and the lytA probe was JOE-labelled. Primer and probe sequences are shown in Table 1. The RT amplification was performed in $25 \mu \mathrm{l}$ reaction volumes containing $2 \times$ TaqMan Universal Master Mix (Applied Biosystems); primers (for S. pneumoniae, Neisseria meningitidis and $H$. influenzae type b) were used at a concentration of $300 \mathrm{nM}$; the FAM-labelled probe was used at a concentration of $25 \mathrm{nM}$ and NEDor JOE-labelled probes were used at a concentration of $50 \mathrm{nM}$. Six microlitres of DNA extract was used for each reaction. All reactions were performed in triplicate. A negative control (no template) and a positive control for each pathogen were included in every run. DNA was amplified in an ABI 7000 sequence detection system (Applied Biosystems) using the following cycling parameters: $95{ }^{\circ} \mathrm{C}$ for $10 \mathrm{~min}$ followed by 45 cycles of a two-stage temperature profile of $95{ }^{\circ} \mathrm{C}$ for $15 \mathrm{~s}$ and $60{ }^{\circ} \mathrm{C}$ for $1 \mathrm{~min}$.

Serotyping. S. pneumoniae serotyping was performed on RT-PCR positive samples through a sequential multiplex PCR on DNA extracted from biological samples as for RT-PCR. Thirty-one primer pairs (Pai et al., 2006; O'Halloran \& Cafferkey, 2005) for serogroups/ serotypes 1, 3, 4, 5, 6A/B, 7F/A, 7C/B, 8, 9V/A, 10A, 11A/D/F, 12A/B/ $\mathrm{F}, 14,15 \mathrm{~A}, 15 \mathrm{~B} / \mathrm{C}, 16 \mathrm{~F}, 17 \mathrm{~F}, 18 \mathrm{~A} / \mathrm{B} / \mathrm{C} / \mathrm{F}$ (two pairs of primers), 19A, $19 \mathrm{~F}, 20,22 \mathrm{~A} / \mathrm{F}, 23 \mathrm{~F}, 31,33 \mathrm{~A} / \mathrm{F}, 34,35 \mathrm{~B}, 35 \mathrm{~F}$ and $38 \mathrm{~F}$ were grouped into nine multiplex reactions. cpsA (pneumococcal capsular polysac-

Table 1. Primer and probe sequences for RT-PCR

\begin{tabular}{|c|c|c|c|}
\hline Gene & Forward primer & Reverse primer & Probe \\
\hline lytA & 5'-ACGCAATCTAGCAGATGAAGC-3' & 5'-TGTTTGGTTGGTTATTCGTGC-3' & $\begin{array}{l}\text { 5'(JOE)-TTTGCCGAAAACGCTTGATACAGGG-6- } \\
\text { carboxytetramethyl-rhodamine (TAMRA)-3' }\end{array}$ \\
\hline $\operatorname{ctr} A$ & 5'-GCTGCGGTAGGTGGTTCAA-3' & 5'-TTGTCGCGGATTTGCAACTA-3' & $\begin{array}{l}\text { 6-FAM- }{ }^{680} \text {-CATTGCCACGTGTCAGCTGCA- } \\
\text { CAT- }{ }^{657} \text {-6-carboxytetramethyl-rhodamine } \\
\text { (TAMRA)-3' }\end{array}$ \\
\hline bexA & 5'-GGCGAAATGGTGCTGGTAA-3 & $\begin{array}{l}\text { 5'-GGCCAAGAGATACTCATAGAA- } \\
\text { CGTT-3' }\end{array}$ & $\begin{array}{l}\text { NED-CACCACTCATCAAACGAATGAGCGTGG-6- } \\
\text { carboxytetramethyl-rhodamine (TAMRA)-3' }\end{array}$ \\
\hline
\end{tabular}


charide synthesis gene) primers (Pai et al., 2006) were included in each reaction mix as a confirmatory test; samples positive for serogroup 14 were also tested for confirmation with a different primer pair (http://www.cdc.gov/ncidod/biotech/strep/pcr.htm). The PCRs were performed in $25 \mu \mathrm{l}$ volumes, with the mixture containing $1 \times$ PCR Master Mix (Qiagen) and primers (0.2-0.5 mM each); $5 \mu \mathrm{l}$ DNA extract was used in each PCR. Amplification was performed in a Perkin-Elmer GeneAmp PCR system 2720 (Applied Biosystems) under the following conditions: $95{ }^{\circ} \mathrm{C}$ for $15 \mathrm{~min}$ followed by 35 amplification cycles of $94{ }^{\circ} \mathrm{C}$ for $30 \mathrm{~s}, 54{ }^{\circ} \mathrm{C}$ for $90 \mathrm{~s}$ and $72{ }^{\circ} \mathrm{C}$ for $60 \mathrm{~s}$. A final hold was performed at $72{ }^{\circ} \mathrm{C}$ for $10 \mathrm{~min}$. As previously described (Kaplan et al., 2004), pneumococcal serotypes were classified as 'vaccine serotype' if they were $4,6 \mathrm{~B}, 9 \mathrm{~V}, 14,18 \mathrm{C}, 19 \mathrm{~F}$ or 23F. They were classified as 'potentially cross-reactive serotypes' if they were from the same serogroups but serotype could not be identified and they were classified as 'non-vaccine serotypes' if samples were positive for serogroups different from those contained in the heptavalent conjugate vaccine.

PCR product detection. The PCR products were analysed by gel electrophoresis on $2 \%$ NuSieve agarose gels (Cambrex Bio Science) in $1 \times$ TAE buffer. Gels were stained with ethidium bromide $(0.5 \mu \mathrm{g}$ $\mathrm{ml}^{-1}$ ), and gel images were recorded. The sizes of the PCR products were determined by comparison with the molecular size standard (100 bp ladder; Novagen).

Microbiology. Blood cultures were performed in BACTEC TM PLUS aerobic bottles (Becton Dickinson). Identification of the pneumococcal isolates was obtained by conventional techniques (Arbique et al., 2004), including optochin susceptibility and bile solubility; serotyping was only performed by molecular methods.

Statistical analysis. All continuous variables were expressed as mean \pm SD. Fisher's exact test, Pearson's chi-square test and the McNemar test were used when appropriate. All $P$ values were twosided, and values less than 0.05 were considered statistically significant. SPSSX version 10.1 was used to analyse data.

\section{RESULTS AND DISCUSSION}

\section{Children with and without IPD}

Ninety-two children [53 males (57.6\%), 39 females $(42.4 \%)$, ratio 1.36 ] were included in the study, 80 with pneumonia, 8 with meningitis/sepsis and 4 with arthritis. Twenty-two IPD cases [12/53 males (22.6\%), 10/39 $(25.6 \%)$ females $(P=\mathrm{NS})]$ were diagnosed in biological samples during the 1-year period by RT-PCR and confirmed by cpsA positivity during the serotyping procedure with multiplex sequential PCR. Blood samples were positive by molecular methods in all 22 patients; $3 / 3$ CSF samples obtained from patients with meningitis and 2/ 2 pleural fluid samples obtained from patients with pneumonia were also positive. The distribution of cases with a different disease is presented in Table 2 . White blood cell count, neutrophil percentage and C-reactive protein did not differ between IPD and non-IPD patients (respectively $19956 \pm 10168$ vs $21447 \pm 10378 P=\mathrm{NS}$; $73.1 \pm 25.6$ vs $76.3 \pm 18.4 P=\mathrm{Ns} ; 16.3 \pm 12.5$ vs $12.6 \pm 10.4$ $P=\mathrm{NS}$; normal value for C-reactive protein is $<0.3 \mathrm{mg}$ $\mathrm{dl}^{-1}$ ). None of the children with IPD had an underlying disease. All were previously healthy. The clinical outcome was evaluated in all 22 cases: no case of death was recorded but one patient suffered a permanent sequela (partial cranial nerves palsy) after pneumococcal meningitis.

\section{Serotyping}

Molecular methods allowed serotyping (Table 3) in 19/22 patients $(86.4 \%)$. The impossibility of serotyping can derive from the presence of serotypes which are not included in the mix used or from a bacterial load below the limit of sensitivity of multiplex PCR. In accord with previously reported data (Corless et al., 2001), in the three patients that could not be serotyped by the multiplex PCR, the cycle number to reach the baseline threshold value in RT-PCR was always higher than 35 , suggesting a very low bacterial load. In patients in whom two biological samples [blood +CFS $(n=3)$ or blood + pleural fluid $(n=2)]$ were available, the same serotype was found in the two different biological samples. Four of the 19 patients had been vaccinated; they had pneumonia due to non-vaccine serotypes. Fifteen children were not vaccinated: 12 (80\%) were infected by vaccine serotypes or by cross-reactive

Table 2. PCR and culture results for Streptococcus pneumoniae in 92 patients

No positivity for other micro-organisms was obtained by cultural methods for any of the samples indicated as negative.

\begin{tabular}{|c|c|c|c|}
\hline & Patients $(n=92)$ & PCR results for S. pneumoniae $n(\%)$ & Culture results for $S$. pneumoniae $n(\%)$ \\
\hline \multirow[t]{2}{*}{ Arthritis } & 4 & Positive 2/4 (50) & Positive 1/4 (25) \\
\hline & & Negative $2 / 4(50)$ & Negative $3 / 4^{*}(75)$ \\
\hline Pneumonia & & Negative $64 / 80(80)$ & Negative $79 / 80(98.75)$ \\
\hline \multirow[t]{2}{*}{ Meningitis/sepsis } & 8 & Positive $4 / 8(50)$ & Positive $2 / 8(25)$ \\
\hline & & Negative $4 / 8(50) \dagger$ & Negative $6 / 8(75) \ddagger$ \\
\hline
\end{tabular}

${ }^{*}$ One was positive for Staphylococcus aureus.

$\dagger$ Four were positive for N. meningitidis.

$\ddagger$ One was positive for $N$. meningitidis. 
Table 3. Serotype distribution in 22 IPD patients according to type of disease and vaccination status

PF, pleural fluid.

\begin{tabular}{|c|c|c|c|c|c|c|c|c|}
\hline & \multirow[t]{2}{*}{ Age (months) } & \multirow[t]{2}{*}{ Vaccinated } & \multirow[t]{2}{*}{ Culture result } & \multicolumn{3}{|c|}{ RT-PCR results (gene lytA) } & \multicolumn{2}{|l|}{ Serotyping } \\
\hline & & & & Blood & CSF & PF & $\begin{array}{l}\text { PCR with serotype- } \\
\text { specific primers }\end{array}$ & $\begin{array}{l}\text { PCR for } \\
\text { gene cps }\end{array}$ \\
\hline \multirow[t]{2}{*}{ Arthritis $(n=2)$} & 6 & No & & POS & & & NT & POS \\
\hline & 10 & No & POS & POS & & & $22 \mathrm{~F}$ & POS \\
\hline \multirow[t]{16}{*}{ Pneumonia $(n=16)$} & 85 & No & & POS & & & 4 & POS \\
\hline & 24 & No & & POS & & POS & $6 \mathrm{~A} / \mathrm{B}$ & POS \\
\hline & 1 & No & & POS & & & $6 \mathrm{~A} / \mathrm{B}$ & POS \\
\hline & 22 & Yes & & POS & & & $19 \mathrm{~A}$ & POS \\
\hline & 22 & No & & POS & & & 8 & POS \\
\hline & 38 & No & & POS & & & 8 & POS \\
\hline & 4 & No & & POS & & & NT & POS \\
\hline & 11 & No & & POS & & & 14 & POS \\
\hline & 70 & No & & POS & & & 18 & POS \\
\hline & 108 & No & POS & POS & & POS & $18 \mathrm{C} \dagger$ & POS \\
\hline & 51 & No & & POS & & & NT & POS \\
\hline & 55 & Yes & & POS & & & 1 & POS \\
\hline & 71 & No & & POS & & & $19 \mathrm{~F}$ & POS \\
\hline & 63 & Yes & & POS & & & 12 & POS \\
\hline & 43 & Yes & & POS & & & 3 & POS \\
\hline & 50 & No & & POS & & & $19 \mathrm{~F}$ & POS \\
\hline \multirow[t]{4}{*}{ Meningitis/sepsis $(n=4)$} & 19 & No & POS & POS & & & 4 & POS \\
\hline & 38 & No & & POS & POS & & $6 \mathrm{~A} / \mathrm{B}$ & POS \\
\hline & 120 & No & POS & POS & POS & & $23 \mathrm{~F}$ & POS \\
\hline & 80 & No & & POS & POS & & $9 \mathrm{~V} / \mathrm{A}$ & POS \\
\hline
\end{tabular}

${ }^{\star}$ NT, Non-typeable.

$\dagger$ Culture confirmed.

serotypes and $3(20 \%)$ were infected by non-vaccine serotypes. All children with major diseases (meningitis/ sepsis) were not vaccinated and were infected by vaccine serotypes or cross-reactive serotypes. At best, cultural methods would have serotyped $4 / 22(18.2 \%)$ patients, thus giving less informative epidemiological data. The use of molecular methods for diagnosis of IPD is well documented (Corless et al., 2001; McAvin et al., 2001; Verhelst et al., 2003; Morrison et al., 2000) but, up to now, pneumococcus typing by molecular methods has been performed only on cultural isolates and not on clinical samples (Pai et al., 2006; Kong et al., 2006). Therefore, the possibility of serotyping was restricted to samples positive in culture. Among the 22 patients with pneumococcal infection diagnosed by molecular methods, 11/22 (2 meningitis, 9 pneumonia) had received antibiotic therapy before hospital admission. The two patients with meningitis had both received a 2 day antibiotic course with cefixime. The nine patients with pneumococcal pneumonia had received antibiotic therapy for a mean duration of 4.11 days (range 1-11 days) with amoxicillin + clavulanic acid (three cases), ceftriaxone (three cases), cefixime (one case), cefixime and teicoplanin (one case). None of the patients who had received antibiotic therapy were positive by cultural methods. Antibiotic therapy is often performed in children with febrile illnesses on an outpatient basis, before hospital admission. For this reason, micro-organism viability may be compromised and cultural methods may be unable to detect invasive pneumococcus infection (Corless et al., 2001; Dalton \& Allison, 1968) or other bacterial infection (Kaczmarski, 1997). On the contrary, PCR-based assays, not requiring viable bacteria, can be successfully used with patients after the beginning of antibiotic therapy. We are aware that positive results of molecular tests in blood may be the results of DNA-aemia and not bacteraemia; however, the association of compatible clinical syndromes with positive results of molecular tests in samples obtained from normally sterile sites (blood, CSF, pleural fluid) appears to strongly suggest an invasive bacterial infection.

\section{Age distribution and incidence of IPD}

The mean age of patients included in the study was $63.1 \pm 45.2$ months (median 52.5 months, range 1-168 
months). The mean age of IPD patients was $45.0 \pm 33.8$ months (median 40.5 months, range 1-120 months) and that of non-IPD patients was $70.3 \pm 47.6$ months (median 60.0 months, range 1-168 months). IPD patients were significantly younger than non-IPD patients $(P=0.01,95 \%$ $\mathrm{CI}=6.8-44.0)$. The age distribution of IPD patients is shown in Fig. 1. The Florentine paediatric population below 14 years of age was 110727 , of which 8963 were below 1 year, 17385 below 2 years of age and 42756 below 5 years of age (Italian National Institute of Statistics, ISTAT, 2007; http://demo.istat.it/). Larger studies are necessary to give a correct estimate of IPD incidence in relation to paediatric age; however, the present data suggest a higher frequency of IPD when evaluated by molecular methods. The incidence rate for IPD obtained by cultural methods was 3.6/100 000 for children below 14 years, 4.7/ 100000 under 5 years, 11.5/100 000 under 2 years and 11.2/ 100000 in the first year of life. Using molecular methods, the incidence rate of IPD in children below 14 years of age was $19.9 / 100000$, the incidence below 5 years of age was $35.1 / 100000$, below 2 years was 51.8/100 000 and below 1 year was $55.8 / 100000$ (Fig. 2). The present data confirm that, as in other countries in the world (D'Ancona et al., 2005; Kaltoft et al., 2000; von Kries et al., 2000; Black et al., 2004), the highest incidence of IPD is found in children below 2 years of age and it progressively declines with increasing paediatric age. IPD incidence in children below 2 years obtained in the present study by cultural methods is similar to data previously reported in Italy (D'Ancona et al., 2005) and in other countries in the world (Gendrel et al., 1997; Juvén et al., 2000; Esposito et al., 2004). The present study suggests that IPD incidence in the paediatric population of the Florentine area, evaluated by molecular methods, appears significantly higher, exceeding 50/ 100000 in children below 2 years and even higher in children in the first year of life. As demonstrated by Rodríguez-Créixems et al. (2003), the incidence of invasive pneumococcal infection is probably even higher than that shown by present results. Indeed patients with fever, often associated with pneumococcal occult bacteraemia (Carvalho et al., 2007), have not been included. Moreover, the present study evaluated only patients admitted to hospital, while patients with pneumonia are usually followed on an outpatient basis. Therefore, the present data are complementary to those obtained by family paediatricians. The main aim of the present study was to evaluate the incidence of invasive pneumococcal infection in the paediatric population, so no data on other aetiological agents responsible for pneumonia cases were collected. The IPD incidence relative to the different diseases studied was 3.6/100 000 for meningitis/sepsis, 1.8/ 100000 for arthritis and 14.4/100000 for pneumonia in children below 14 years of age (Fig. 2).

\section{PCR sensitivity}

None of the patients negative for pneumococcus by RTPCR were positive by cultural methods; among the 22 patients who were positive for pneumococcus by molecular methods, $4 / 22(18.2 \%)$ were also positive by cultural methods (Table 2). RT-PCR appeared significantly more sensitive than culture in diagnosing IPD (Cohen's $\kappa$ 0.41; McNemar $P=0.000008$ ). The four $S$. pneumoniae isolates were penicillin-susceptible. For 18/22 (81.8\%) patients, diagnosis of IPD was only obtained by RT-PCR. In particular, among patients with pneumococcal meningitis or sepsis, $2 / 4(50.0 \%)$ were only positive by RT-PCR, while among patients with pneumococcal pneumonia, 15/16 $(93.8 \%)$ were only positive by RT-PCR. Of the two patients with arthritis who were positive for S. pneumoniae by RT-PCR, one was positive by cultural methods. Increased sensitivity of molecular methods is even more evident in patients with pneumonia than in patients with meningitis/sepsis. For meningitis, the molecular test routinely performed in our laboratory can diagnose both S. pneumoniae and N. meningitidis, and 8/8 meningitis/

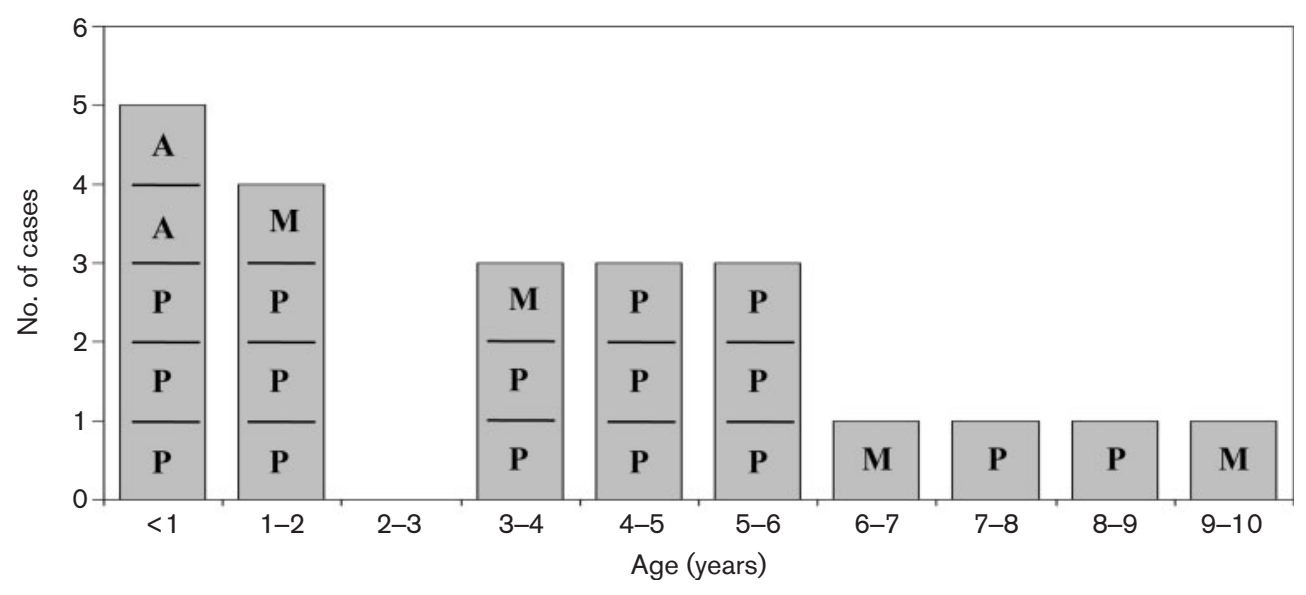

Fig. 1. Distribution of 22 cases of IPD according to age and type of disease. $A$, Arthritis; $M$, meningitis; $P$, pneumonia. 


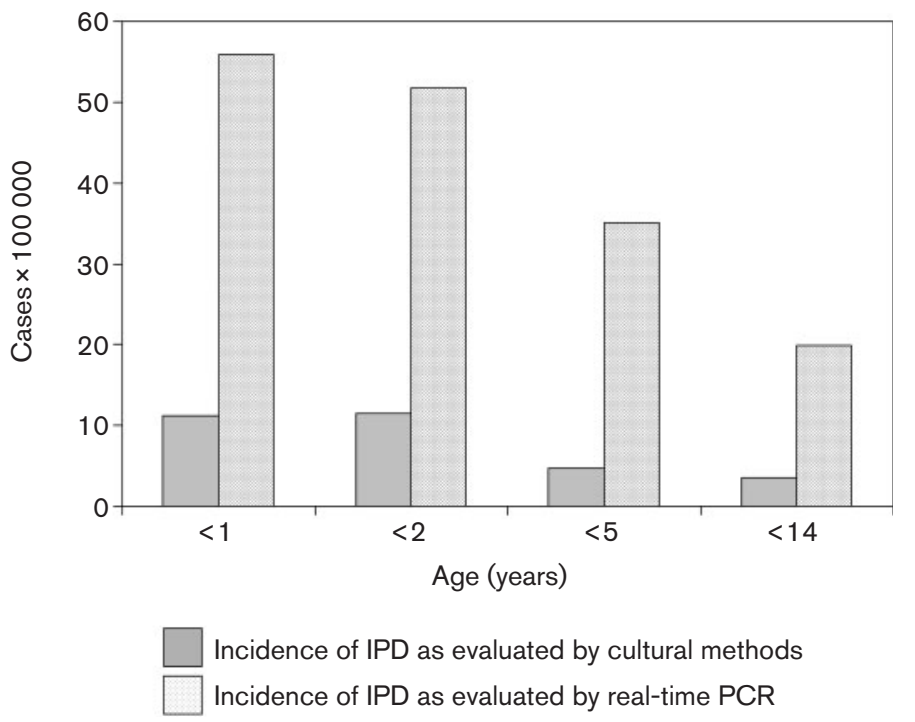

Fig. 2. Incidence of IPD as evaluated by cultural or molecular methods.

sepsis cases could be identified as due to those microorganisms. The sensitivity of RT-PCR is double that of culture in the diagnosis of meningitis/sepsis and it is about ten times higher in patients with pneumonia. The difference between the two groups of patients can be explained in two ways: (i) patients with meningitis and sepsis usually have a much higher bacterial load, as demonstrated by RT-PCR (data not shown), so that cultural methods, though less sensitive, can detect infection; (ii) patients with meningitis/sepsis usually have a rapid progression of the disease, becoming severe in a few hours, so it is less probable that they have received prolonged antibiotic therapy before hospital admission.

RT-PCR and multiplex sequential PCR are very sensitive methods and their use on clinical samples can help in obtaining a closer estimate of the real incidence of IPD. However, when a positive result is obtained in samples considered negative by the established 'gold standard' (culture from blood or CSF), it might be speculated that this is due to poor specificity of PCR rather than improved sensitivity. For this reason, for every sample, we used two different targets from two unrelated genes, lytA (known to be extremely sensitive and specific) (Carvalho et al., 2007) in RT-PCR and cps in sequential multiplex PCR. Positive results from both targets in the same sample enhance confidence in the sample positivity. Moreover, more than $80 \%$ of the samples could also be serotyped using specific primers for each serotype, different from those for $c p s$ and lytA. At present, cultural methods remain absolutely necessary. First, cultural methods can give important data on antibiotic resistance and individual pathogens which are not included in molecular protocols. Moreover, in some cases, the molecular methods described in the present paper cannot distinguish between serotypes within the same serogroup (i.e. 6A/6B) and more expensive and timeconsuming molecular tests would be necessary (Pai et al.,
2005). Even if discriminating between serotypes may sometimes be irrelevant for a clinical approach, because a vaccine-induced cross-protection is well known (Vakevainen et al., 2001), distinguishing between serotypes is undoubtedly useful for correct epidemiological evaluation.

At present, case definition of IPD in many countries is based on positive results of standard cultural methods (Pebody et al., 2006). Given that, in many cases, molecular methods show a higher sensitivity (Corless et al., 2001; Van Gastel et al., 2007; Lahti et al., 2006), it could be suggested that case definition should also include diagnosis performed by molecular methods. Indeed, inclusion of molecular assays in the diagnostic tests would improve the diagnostic ability and case ascertainment of invasive bacterial infections.

In conclusion, even though larger studies are required to confirm the present results on IPD incidence, this study demonstrates, for the first time, that an association between RT-PCR and multiplex sequential PCR can be used directly on biological samples for diagnosis and serotyping of IPD. It is likely that similar methods can be used to develop PCR assays able to detect all clinically relevant pneumococcal serotypes that may be found in IPD. The method will be helpful in epidemiological evaluation of circulating serotypes and, consequently, in making correct decisions about vaccination. Molecular methods will also be helpful, even after mass vaccination starts, in monitoring possible epidemiological changes in serotype distribution.

\section{ACKNOWLEDGEMENTS}

We are grateful to Professor Gianni Pozzi from the University of Siena for his help in discussing methods and results. 


\section{REFERENCES}

Arbique, J. C., Poyart, C., Trieu-Cuot, P., Quesne, G., Carvalho, Mda, G., Steigerwalt, A. G., Morey, R. E., Jackson, D., Davidson, R. J. \& Facklam, R. R. (2004). Accuracy of phenotypic and genotypic testing for identification of Streptococcus pneumoniae and description of Streptococcus pseudopneumoniae sp. nov. J Clin Microbiol 42, 4686-4696.

Black, S., Shinefield, H., Baxter, R., Austrian, R., Bracken, L., Hansen, J., Lewis, E. \& Fireman, B. (2004). Postlicensure surveillance for pneumococcal invasive disease after use of heptavalent pneumococcal conjugate vaccine in Northern California Kaiser Permanente. Pediatr Infect Dis J 23, 485-489.

Black, S., Shinefield, H., Eskola, J. \& Whitney, C. (2007). Pneumococcal conjugate vaccine. In Vaccines, V edn, chapter 23. Edited by S. A. Plotkin, W. A. Orenstein \& P. Offit. Philadelphia, PA: WB Saunders.

Carvalho, Mda, G., Tondella, M. L., McCaustland, K., Weidlich, L., McGee, L., Mayer, L. W., Steigerwalt, A., Whaley, M., Facklam, R. R. \& other authors (2007). Evaluation and improvement of real-time PCR assays targeting $l y t, p l y$, and $p s a A$ genes for detection of pneumococcal DNA. J Clin Microbiol 45, 2460-2466.

CDC (1997). Prevention of pneumococcal disease: recommendations of the Advisory Committee on Immunization Practices (ACIP). MMWR Recomm Rep 46, 1-24.

Clarke, S. C. (2006). Detection of Neisseria meningitidis, Streptococcus pneumoniae, and Haemophilus influenzae in blood and cerebrospinal fluid using fluorescence-based PCR. Methods Mol Biol 345, 69-77.

Corless, C. E., Guiver, M., Borrow, R., Edwards-Jones, V., Fox, A. J. \& Kaczmarski, E. B. (2001). Simultaneous detection of Neisseria meningitidis, Haemophilus influenzae, and Streptococcus pneumoniae in suspected cases of meningitis and septicemia using real-time PCR. J Clin Microbiol 39, 1553-1558.

Dalton, H. P. \& Allison, M. J. (1968). Modification of laboratory results by partial treatment of bacterial meningitis. Am J Clin Pathol 49, 410-413.

D’Ancona, F., Salmaso, S., Barale, A., Boccia, D., Lopalco, P. L., Rizzo, C., Monaco, M., Massari, M., Demicheli, V. \& other authors (2005). Incidence of vaccine preventable pneumococcal invasive infections and blood culture practices in Italy. Vaccine 23, 24942500

Domínguez, A., Salleras, L., Cardeñosa, N., Ciruela, P., Carmona, G., Martínez, A., Torner, N. \& Fuentes, M. (2002). The epidemiology of invasive Streptococcus pneumoniae disease in Catalonia (Spain). A hospital-based study. Vaccine 20, 2989-2994.

Esposito, S., Bosis, S., Colombo, R., Carlucci, P., Faelli, N., Fossali, E. \& Principi, N. (2004). Evaluation of rapid assay for detection of Streptococcus pneumoniae urinary antigen among infants and young children with possibile invasive pneumococcal disease. Pediatr Infect Dis J 23, 365-367.

Gendrel, D., Raymond, J., Moulin, F., Iniguez, J. L., Ravilly, S., Habib, F., Lebon, P. \& Kalifa, G. (1997). Etiology and response to antibiotic therapy of community-acquired pneumonia in French children. Eur $J$ Clin Microbiol Infect Dis 16, 388-391.

Hausdorff, W. P., Bryant, J., Paradiso, P. R. \& Siber, G. R. (2000). Which pneumococcal serogroups cause the most invasive disease: implications for conjugate vaccine formulation and use, part I. Clin Infect Dis 30, 100-121.

Juvén, T., Mertsola, J., Waris, M., Leinonen, M., Meurman, O., Roivainen, M., Eskola, J., Saikku, P. \& Ruuskanen, O. (2000). Etiology of community-acquired pneumonia in 254 hospitalized children. Pediatr Infect Dis J 19, 293-298.
Kaczmarski, E. B. (1997). Meningococcal disease in England and Wales: 1995. Commun Dis Rep CDR Rev 7, R55-R59.

Kaltoft, H., Zeuthen, N. \& Konradsen, H. B. (2000). Epidemiology of invasive pneumococcal infections in children aged 0-6 years in Denmark: a 19-year nationwide surveillance study. Acta Paediatr Suppl 89, 3-10.

Kaplan, S. L. (2005). Osteomyelitis in children. Infect Dis Clin North Am 19, 787-797.

Kaplan, S. L., Mason, E. O., Jr, Wald, E. R., Schutze, G. E., Bradley, J. S., Tan, T. Q., Hoffman, J. A., Givner, L. B., Yogev, R. \& Barson, W. J. (2004). Decrease of invasive pneumococcal infections in children among 8 children's hospitals in the United States after the introduction of the 7-valent pneumococcal conjugate vaccine. Pediatrics 113, 443-449.

Kong, F., Brown, M., Sabananthan, A., Zeng, X. \& Gilbert, G. L. (2006). Multiplex PCR-based reverse line blot hybridization assay to identify 23 Streptococcus pneumoniae polysaccharide vaccine serotypes. J Clin Microbiol 44, 1887-1891.

Lahti, E., Mertsola, J., Kontiokari, T., Eerola, E., Ruuskanen, O. \& Jalava, J. (2006). Pneumolysin polymerase chain reaction for diagnosis of pneumococcal pneumonia and empyema in children. Eur J Clin Microbiol Infect Dis 25, 783-789.

McAvin, J. C., Reilly, P. A., Roudabush, R. M., Barnes, W. J., Salmen, A., Jackson, G. W., Beninga, K. K., Astorga, A., McCleskey, F. K. \& other authors (2001). Sensitive and specific method for rapid identification of Streptococcus pneumoniae using real-time fluorescence PCR. J Clin Microbiol 39, 3446-3451.

Morrison, K. E., Lake, D., Crook, J., Carlone, G. M., Ades, E., Facklam, R. \& Sampson, J. S. (2000). Confirmation of $p s a A$ in all 90 serotypes of Streptococcus pneumoniae by PCR and potential of this assay for identification and diagnosis. J Clin Microbiol 38, 434-437.

O'Halloran, D. M. \& Cafferkey, M. T. (2005). Multiplex PCR for identification of seven Streptococcus pneumoniae serotypes targeted by a 7-valent conjugate vaccine. J Clin Microbiol 43, 3487-3490.

Ostapchuk, M., Roberts, D. M. \& Haddy, R. (2004). Communityacquired pneumonia in infants and children. Am Fam Physician 70, 899-908.

Overturf, G. D. (2005). Defining bacterial meningitis and other infections of the central nervous system. Pediatr Crit Care Med 6, S14-S18.

Pai, R., Limor, J. \& Beall, B. (2005). Use of pyrosequencing to differentiate Streptococcus pneumoniae serotypes 6A and 6B. J Clin Microbiol 43, 4820-4822.

Pai, R., Gertz, R. E. \& Beall, B. (2006). Sequential multiplex PCR approach for determining capsular serotypes of Streptococcus pneumoniae isolates. J Clin Microbiol 44, 124-131.

Pebody, R. G., Hellenbrand, W., D’Ancona, F., Ruutu, P. \& European Union funded Pnc-EURO contributing group (2006). Pneumococcal disease surveillance in Europe. Euro Surveill 11, 171-178.

Rodríguez-Créixems, M., Bertó, J., Muñoz, P., Alonso, R., Alcalá, L. \& Bouza, E. (2003). Increased incidence of pneumococcal bloodstream infections. Pediatr Infect Dis J 22, 661-663.

Russell, J. A. (2006). Management of sepsis. N Engl J Med 355, 16991713.

Smith, M. D., Stuart, J., Andrews, N. J., Telfer Brunton, W. A. \& Cartwright, K. A. (1998). Invasive pneumococcal infection in South and West England. Epidemiol Infect 120, 117-123.

Vakevainen, M., Eklund, C., Eskola, J. \& Kayhty, H. (2001). Crossreactivity of antibodies to type $6 \mathrm{~B}$ and $6 \mathrm{~A}$ polysaccharides of Streptococcus pneumoniae, evoked by pneumococcal conjugate vaccines, in infants. J Infect Dis 184, 789-793. 
Van Gastel, E., Bruynseels, P., Verstrepen, W. \& Mertens, A. (2007). Evaluation of a real-time polymerase chain reaction assay for the diagnosis of pneumococcal and meningococcal meningitis in a tertiary care hospital. Eur J Clin Microbiol Infect Dis 26, 651-653.

Verhelst, R., Kaijalainen, T., De Baere, T., Verschraegen, G., Claeys, G., Van Simaey, L., De Ganck, C. \& Vaneechoutte, M. (2003). Comparison of five genotypic techniques for identification of optochin-resistant pneumococcus-like isolates. J Clin Microbiol 41, 3521-3525. von Kries, R., Siedler, A., Schmitt, H. J. \& Reinert, R. R. (2000). Proportion of invasive pneumococcal infections in German children preventable by pneumococcal conjugate vaccines. Clin Infect Dis 31, 482-487.

Whitney, C. G., Farley, M. M., Hadler, J., Harrison, L. H., Bennett, N. M., Lynfield, R., Reingold, A., Cieslak, P. R., Pilishvili, T. \& other authors (2003). Active Bacterial Core Surveillance of the Emerging Infections Program Network. Decline in invasive pneumococcal disease after the introduction of protein polysaccharide conjugate vaccine. N Engl J Med 348, 1737-1746. 